\title{
The application of ESI maps with the GIS technique to coastal oil-spill cleanups in Taiwan
}

\author{
Y.-C. Chen \& L.-J. O’Yang \\ Department of Environmental Engineering, \\ Da-Yeh University, Taiwan
}

\begin{abstract}
Fragile ecosystems and the harnessing of resources for human use are two major elements that have to coexist in coastal regions. The sensitivity of the coastal environment to oil spills and related cleanups depends on the presence of these resources, and can be assessed by the level of potential impact that would be caused by an oil spill. In this study, the Environmental Sensitivity Index (ESI) and maps associated with it (ESI maps) are introduced and will be applied to the Taiwan coastline. The ESI indicators include shoreline classification, biological resources and resources for human use (socio-economic resources).

These indicators will represent the extent to which a specific coastal area would be vulnerable to the effects of an oil spill. In other words, these indicators tell us the level of reactions induced as a result of the adverse changes in safety, survival and reproduction in ecosystems.

A framework for an index setup procedure will be described and constructed in this study. ESI maps are very useful tools as complementary guidelines for decision-making support systems in oil-spill contingency plans. Furthermore, electronic ESI maps combined with a query scheme in a geographic information system could be convenient to apply and use.
\end{abstract}

Keywords: oil spills, environmental sensitivity index (ESI), ESI maps, geographic information system (GIS). 


\section{Introduction}

In 2001, the Lungken environmentally protected area inside Kenting National Park in Taiwan was polluted by an oil-spill accident from a grounded boat. Precious coral-reef area was in danger from the oil as well as an iron ore covering. Hesitant response and improper cleanups actually damaged the sensitive coral coastline and the biota on it. The sensitivity of coastal environments to spilled oil and its cleanup reveals the function of the natural environment and the development of such areas for human-use resources where they overlap in coastal areas. Because of this, the information indicating shoreline classification, biological resources and human-use resources is important in order to assess the sensitivity accordingly. More importantly, this information can also be applied to oil-spill emergency responses with proper cleanups.

Through this study, the Environmental Sensitivity Index (ESI) and corresponding maps originally created in United States are introduced and applied to the Taiwan coastline. A framework for an index setup procedure is also described and constructed. Furthermore, the electronic ESI maps combined with a query scheme in geographic information systems are convenient to apply and use.

\section{Environmental sensitivity}

\subsection{Environmentally sensitive areas}

Environmentally Sensitive Areas (called ESAs, or critical areas) are those requiring special management to protect important scenery, wildlife, places of historical and cultural interest, and other natural systems or processes (Newman and Steiner [1]). This means that a well-defined plan involving policy statements and map indications is required to indicate any restrictions on the use of land as a result of hazardous conditions or because it is environmentally sensitive to development. ESAs are usually associated with valuable ecosystems, wildlife habitats or areas of natural hazards. The New Jersey Pinelands Commission [2] identifies critical areas that include: ecologically critical areas, perceptual and cultural critical areas, resource production critical areas, and natural hazard critical areas. Moreover, thirteen kinds of critical areas are refined and managed depending on their characteristics.

In Taiwan, there are several protection or conservation areas which could be defined as critical areas designated under special legislation, e.g. The National Park Act, The Cultural Property Conservation Act, The Wildlife Conservation Act, and The Coastal Nature Environment Protection Plans, etc. Nevertheless, the operations or management for those critical areas still cannot supply enough background information to construct a contingency plan for oil spills. A lack of detailed information makes it difficult to recognize the vulnerability of coastline or the sensitivity of the resources on it. Therefore, the need for an ESI and the affiliated maps is extremely urgent. 


\subsection{Environmental sensitivity index and maps}

Since 1979, ESI and the ESI maps have been established as an integral part of oil-spill contingency plans and emergency responses in the United States. Similar ideas have been applied in many other countries, e.g. Canada, Denmark, Japan, and Thailand etc., for emergency response to oil spills or pollutants as well as coastal resources management. Since 1989, GIS techniques have been able to transform traditional paper maps into electronic ones [3]. Digital databases will expand ESI maps' applications and utilizations NOAA.

ESI maps that serve as quick references for oil-spill response-teams and managers of coastal zones are also useful for identifying sensitive resources before a spill occurs. In this way, protection priorities could be identified and established, and cleanup strategies could be designed in advance.

There are three types of information included in ESI maps: shoreline classification, biological resources and human-use resources. They are delineated on maps by standardized colour-coding, symbols, or other markings as shown in fig. 1 for clear classifications like shoreline types, protected biota and sensitive areas, etc. ESI maps could show the natural presence of oil, undisturbed habitat, and resources, which are important to humans that would be particularly sensitive to oil spills, as well as the expected ease of cleanup according to an area's vulnerability.

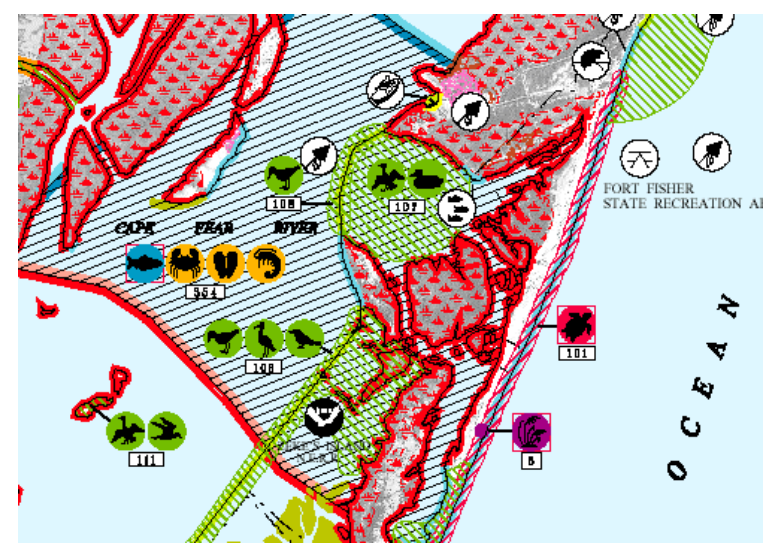

Figure 1: Example of an ESI map prepared by NOAA.

\section{Setup of index}

\subsection{Shoreline classification}

Inter-tidal habitats are at risk during spills because of the high likelihood of being directly polluted when floating slicks impact on the shoreline as well as improper cleanups. Oil pollution varies significantly depending on shoreline type, and proper cleanup methods are shoreline-specific. The sensitivity of a 
particular habitat on a coastline is measured by: (1) shoreline type (substrate, grain size, tidal elevation, origin); (2) exposure to wave and tidal energy; (3) analysis of the natural presence of oil on the shoreline; (4) biological productivity and sensitivity; (5) ease of cleanup without causing more harm. Therefore, ESI shoreline rankings from 1 to 10 representing low to high sensitivity have been defined based on specified criteria by NOAA [3].

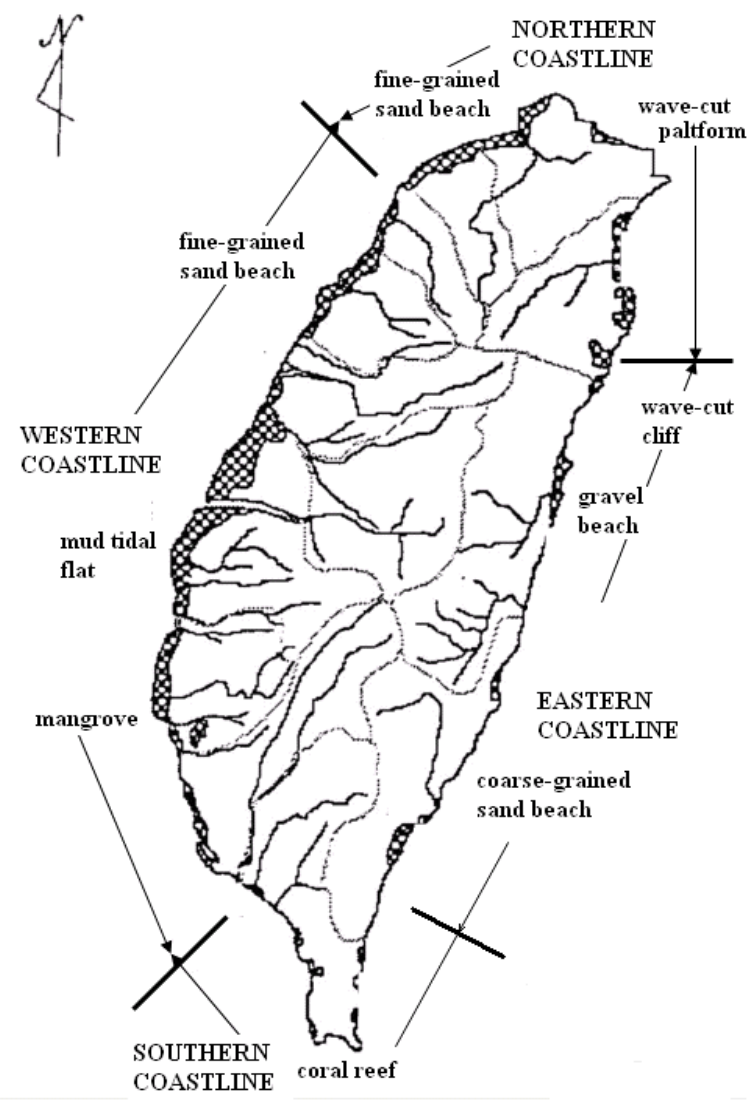

Figure 2: Geomorphic classifications of shoreline in Taiwan.

The major geomorphic classifications of Taiwanese shoreline are: exposed mud tidal flat, fine-grained sandy beach, wave-cut platform, and coral reef. These are shown in their specific locations in fig. 2. The sensitivity to oil spills for these coastal environments are characterized below according to descriptions from Michel and Hayes [4]:

(1) Exposed tidal flats (ESI rank =7): Very low sloping flats are covered by deposits of mud or sand from river sediment when exposed at low tide. There are very wide flats of several kilometers located on the macro-tidal area on the western coastline of Taiwan. In this environment, would not 
usually adhere to the surface of the flats, but would rather move across and accumulate at the high-tide line. Oil does not penetrate the water-saturated sediment. The sediment is very soft and inherently bad at supporting heavier traffic for cleanup. However, tidal currents draining off the wide flats could generate enough energy to lift the oil crust and mobilize the sediment. It could provide a so-called "self-cleansing" mechanism. Biological impacts could be severe, such as primarily the suffocation of fauna (i.e. aquatic animals living in the substrate), since biological utilization can be very high, with varying numbers of bivalves, macro-invertebrates, and polychaetes, etc.

(2) Fine-grained sandy beach (ESI rank $=3$ ): Sandy beach, mostly located on the northwestern coastline of Taiwan, is an accumulation of unconsolidated sediment transported by wave-generated motion. Maximum penetration of oil into fine-grained sand will be less than $10 \mathrm{~cm}$ and burial of the oiled layer along the upper beach face by clean sand will be less than $30 \mathrm{~cm}$ within the first few weeks after the spill. Hard, compact sediments could support pedestrian and vehicular traffic for an easy cleanup. Biological impacts may not be severe due to lower fauna populations on it even if suffocation happened.

(3) Wave-cut platform (ESI rank = 2): Platform is located on the northern coastline of Taiwan and composed of a rock bench with an irregular surface and tidal pools on it. Oil will not adhere to the rock platform, but will rather be transported across the platform and accumulate along the high-tide line. Exposed to high wave energy, cleanup is usually not even necessary. However, smothering or exposure to the toxic parts of fresh oil would probably kill living organisms inside the tidal pools. Also, sea birds using the platforms as haulouts may get oil on their feathers, which would induce hypothermia and a loss of water-repellence.

(4) Coral reefs $(E S I$ rank $=10)$ : Most fringing reefs are located within a small part of Taiwan's southern coastline. Although coral reefs are mostly subtidal in nature, they are susceptible to stress from sediment and chronic oil pollution dissolved in the water. Higher risk may happen in inter-tidal reefs and reef flats, where direct contact with the oil is likely or where leaching from adjacent areas creates a chronic source of oil exposure.

\subsection{Biological resources}

Many species of biota that are vulnerable to oil have wide-ranging habitats, and may show up over large areas at any one time. However, they can be especially sensitive at particular times and places. Sensitivity is determined by the areas at risk from oil spills, for example if: (1) many individuals are concentrated in a small area; (2) early life stages are present in certain areas; (3) specific life stages or important for migration; (4) specific areas for propagation of species; (5) threatened or endangered species; (6) a substantial percentage of an animal or plant population is likely to be exposed to oil (NOAA [4]).

When considering the resources at risk in a given area, seasonal migrants as well as resident populations should be included. Also, the life stages present during any given season will help in determining the sensitivity of different 
populations. In fact, sensitivity to oily toxins varies greatly by species, by life stage within a particular species, and by individual. The only acute effect of oil on shoreline organisms especially such as inter-tidal invertebrates and some plants may be the physical process of smothering. On the other hand, chronic (sometimes called sub-lethal) effects can impact on an organism's physiology, behavior, or reproductive capability. However, chronic effects are harder to detect than acute effects and may require more intensive studies conducted over a longer period of time. In addition to this, some ecological effects that alter predator-prey interaction may result in changes in relative numbers of a species' composition in an area (Scholz, et al [5]).

Also, the impact of oil on species groups varies. A broad categorization could be made for the anticipated degree of impact as low, medium and high level, as shown in table 1 (NAS [6]). Because of the presence of a large population of wildlife at the exposed tidal flat on the Western coastline of Taiwan, impacts can be significant as highly sensitive fauna, e.g. benthic invertebrates, at risk of water-accommodated fraction or being smothered by oil. The ecological effects thereby also impact on birds and other predators by reducing food sources.

Table 1: Proposed degree of impact and its sensitivity to biological resources.

\begin{tabular}{|l|l|c|}
\hline \multicolumn{1}{|c|}{ Level of impact } & \multicolumn{1}{|c|}{ Representative biota } & Sensitivity \\
\hline $\begin{array}{l}\text { Little or no long-term } \\
\text { effects }\end{array}$ & $\begin{array}{l}\text { Annelids, gastropods, } \\
\text { copepods }\end{array}$ & Low \\
\hline Some effects & Macrophytes, barnacles & Medium \\
\hline Long-term effects: & $\begin{array}{l}\text { Corals, bivalves, decapod } \\
\text { crustacea }\end{array}$ & High \\
\hline
\end{tabular}

\subsection{Human-use resources}

Four major categories of human-use resources (or so-called socio-economic resources) are more vulnerable to damage from oil spills. They are: (1) high-use recreational and shoreline access areas, like marinas, recreational beaches, and ferries; (2) designated natural resource management or protected areas, like national parks, wildlife refuges, preserves, and reserves; (3) resource extraction sites and aquaculture sites like mining leases, water intakes, and oyster aquafarms; (4) water-associated archaeological, historical, and cultural sites (NOAA [3]).

Since the impact of oil on these kinds of resources is not so direct and obvious, the sensitivity of human-use resources is hard to measure clearly. The marking of "yes" or "no" by using symbols or markings will make it easier to distinguish the sensitivity. Also, the seasonal activity of socio-economic resources such as recreational beaches or aquaculture sites has to be considered for differential sensitivity. On the Western coastline of Taiwan, while the raising season of oyster aquafarms begins in Summer at exposed tidal flats, the fishing economy could be brought to a standstill by spilled oil. In addition to this, many kinds of 
recreational or leisure activities, including fishing, boating, wildlife viewing, and sight-seeing would also have to be limited.

\section{GIS application on ESI maps}

\subsection{The contents of ESI-GIS map}

An ESI map is a digital map-based site. It could be incorporated with GIS as a decision-support system (DSS) for emergency response to oil-spill cleanups. The query application was designed through Microsoft Windows, Microsoft Access, and the ESRI ArcView software. Users can select specific elements, such as colour-coding, symbols, or other markings on the map, which display spatial data on coastline description, biological resources and habitat as well as important resources or facilities for human use based on their vulnerability to oil. The hatched symbols on a specific site also provide a large amount of text-based background information (also called implicit attribute data) for each individual displayed resource within the region concerned. Detailed information on species distribution and characteristics of their habitats is also included.

The major system of GIS application can be divided into two parts, as shown by the demo module and query module in fig. 3. GIS-ESI maps provide simple selection-by-pointing query capabilities and display their functions by using object-oriented, visual, and user-friendly SQL techniques. The basic query in this system is point-query, which essentially exhibits implicit information at a position specified by the user when they click directly on a symbol or marking on the map. A regional query is also provided, which encloses hatched symbols and information about places that are contained in, adjacent to, or overlap the hatched area. The demo module responding to a query will display the attribute data and ESI contents for a specified point or region. Therefore, GIS-ESI could be used for the following purposes: (1) as a tool for quickly searching sensitive areas which could potentially be damaged by current or a oil spill; (2) as an indicator for how to protect the selected species and their habitats; (3) as a useful resource detailing the proper skills and available facilities to serve cleanups for oil spills. The American Petroleum Institute (API) cooperating with other government agents (e.g. EPA, NOAA, and the Coast Guard) has developed a set of clean-up skills with 28 kinds of processes considering proper timing, suitable situations and biological limitations (SEA [7]).

An example showing the explicit symbol (marking, etc. on a map) and implicit data (a pop-up window that displays attribute data for a specified ESI symbol) is shown in fig. 4 for a section of Taiwanese coastline. In this area a wide tidal flat is illustrated as an oyster aquafarm and a precious waterfowl habitat. Fauna, is also rich within this muddy intertidal zone. This kind of coastline is given a vulnerability rating of medium to high (ESI rank $=7$ ) and the biological risk is presumed to be high for excavating oil. On top of this, the most sensitive socioeconomic resource would be the high number of aquafarms with the large amounts of oyster. 


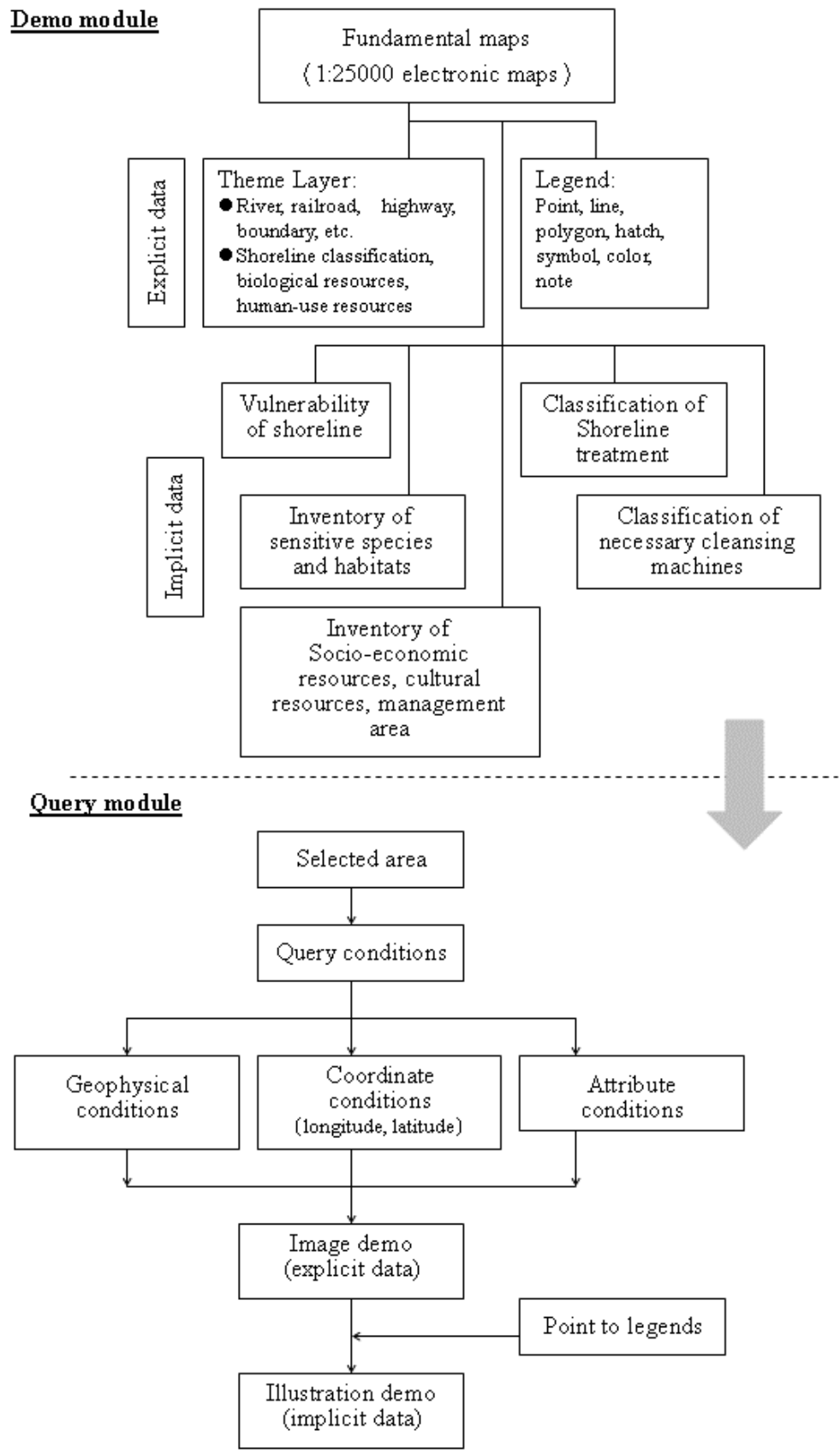

Figure 3: Illustrates class hierarchy of demo and query modules for GIS-ESI maps. 


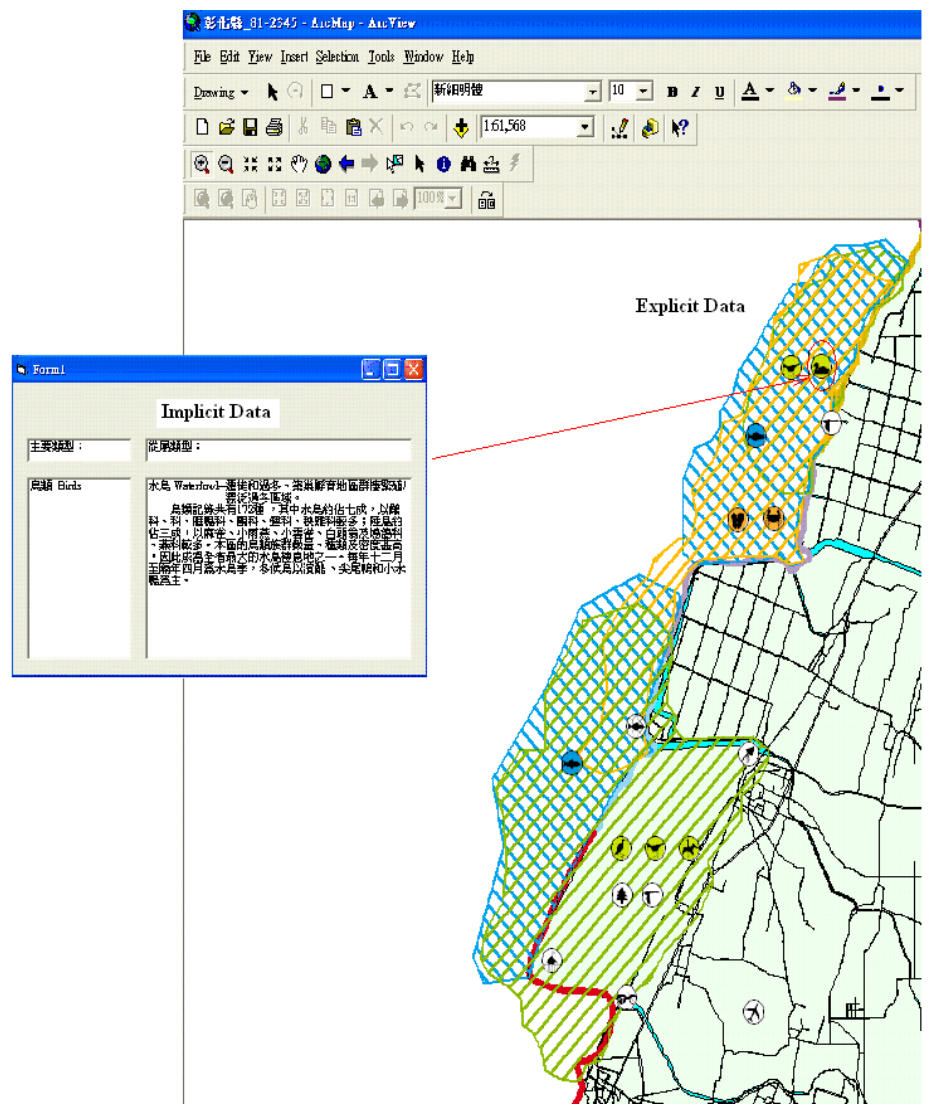

Figure 4: Example of an ESI map showing the Changhua coastline in Taiwan.

\subsection{The role of ESI maps in emergency response}

Oil spill contingency planning and response is a complex and challenging crossdisciplinary activity. Effective oil-spill response planning demands access to a large amount of available data and information. The ability to rapidly process and manage this important information is also vital. The various windows of opportunity need to be specified for effective utilization of coastal oil spill response technologies and methodologies, especially in clean-up operations (Nordvik [8]).

Normally, the standard processes for emergency response (in order) are: notification, evaluation, response, cleanup and monitoring. A predefined and prescribed ESI map can provide detailed and clear information, which plays an important role in contingency planning. Before the commander decides the correct priority of response within a very short amount of time, the following questions need to be considered in advance: 
(1) Where is the most sensitive section of shoreline?

(2) Which areas can and must be protected?

(3) How can we apply the proper skills or technology to the cleanup?

(4) What kind of heavy machinery or clean-up tools are not suitable to use?

If there is a pre-existing and well-considered decision-making support system that can be used, the minimum damage can be controlled and the risk can be lowered. ESI maps should play an important role in the emergency response to oil spills.

\section{Summary}

The most concerning aspects of oil spills are the impacts induced by oil pollutants as well as by the clean-up processes. These influences on the natural ecosystems and human socio-economic resources have been seriously considered. In fact, ESI maps are very effective tool in an oil spill contingency plan. They can identify the sensitive areas and suggest suitable clean-up methods to minimise serious negative impacts. Also, a query scheme using GIS is possible when applied to combine with the electronic maps for use together with a decision support system. Through more widely accessible storage space in computers, the integration, transmission and web-sharing of data will enable us to reduce risks to a minimum.

\section{Acknowledgement}

This paper is part of the results of a research project under contract ORD-9406 from Da-Yeh University in Taiwan. Any support with funding is appreciated by the authors.

\section{References}

[1] Newman, H.G. \& Steiner, F.R., The Definition of Environmentally Sensitive Areas for Landscape Planning, Scientific Paper No. 6360, Pullman, WA: College of Agriculture Research Centre, Washington State University, 1983.

[2] New Jersey Pinelands Commission, Critical Areas Study for the Pinelands, Philadelphia, PA: Golden and Halpern, 1980.

[3] NOAA, Environmental Sensitivity Index Guidelines, NOAA Technical Memorandum NOS OR\&R 11, Seattle, Washington, 2002.

[4] Michel, J. \& Hayes, M.O., Sensitivity of coastal environments to oil, in Introduction to Coastal Habitats and Biological Resources for Oil Spill Response, Chapter 3, NOAA Report No. HMRAD 92-4, 1992.

[5] Scholz, D.; Michel, J.; Shigenaka, G. \& Hoff, R. Biological resources, in Introduction to Coastal Habitats and Biological Resources for Oil Spill Response, Chapter 4, NOAA Report No. HMRAD 92-4, 1992.

[6] National Academy of Sciences (NAS) Oil in the Sea: Inputs, fates and Effects, National Academy Press, Washington, D.C., 1985. 
[7] Scientific and Environmental Associates, Inc. (SEA). Characteristics of Response Strategies: A Guide for Spill Response Planning in Marine Environments. Prepared for the Marine Manual Update Workgroup, American Petroleum Institute (API), Washington, D.C., 78 p., 2001.

[8] Nordvik, A.B. Time window-of-opportunity strategies for oil spill planning and response, Pure Appl. Chem., 71(1), pp. 5-16, 1999. 